\title{
Dynamic tracking error with shortfall control using stochastic programming
}

\author{
Diana Barro Elio Canestrelli
}

\begin{abstract}
In this contribution we tackle the issue of portfolio management combining benchmarking and risk control. We propose a dynamic tracking error problem and we consider the problem of monitoring at discrete points the shortfalls of the portfolio below a set of given reference levels of wealth. We formulate and solve the resulting dynamic optimization problem using stochastic programming. The proposed model allows for a great flexibility in the combination of the tracking goal and the downside risk protection. We provide the results of out-of-sample simulation experiments, on real data, for different portfolio configurations and different market conditions.
\end{abstract}

Keywords: dynamic portfolio optimization, tracking error, shortfall.

M.S.C. classification. 90C15, 90B50, 90C 90

J.E.L. classification. C61, C63, G11.

\section{Introduction}

Measuring risk is a crucial issue in financial modeling and it is relevant both for pricing purposes and for asset allocation problems. In particular, investors are concerned with the measurement and the management of risk in such a way that they can obtain a portfolio which is compliant with their risk attitude.

The majority of investors are more concerned with downside risk rather than upside risk, and there is experimental evidence that they treat differently losses from gains. This introduces the need for asymmetric risk measures and can account for

Diana Barro

Dept. of Economics, University Ca' Foscari Venice, Cannaregio 873

30121 Venice Italy and SSAV e-mail: d.barro@unive.it

Elio Canestrelli

Dept. of Economics, University Ca' Foscari Venice, Cannaregio 873

30121 Venice Italy e-mail: canestre@unive.it 
the growing interest of investors in mean return-downside risk portfolio models. The key idea of these approaches is to separate the downside deviations from the upside potential and control only the first part of risk. For a classification of different risk measure in portfolio selection see, for example, [7].

Risk measurement is strictly connected with the definition of a term of comparison with respect to which we can contrast and compare the risk/return profile of our portfolio. Moreover, it is common practice to monitor the performance of a portfolio, or a fund manager, with reference to an explicitly declared, or implicitly assumed, benchmark. This allows for a more objective assessment of the risk profile and the performance evaluation of the investment, linking it with current market conditions.

The tracking error and tracking error volatility are widely used measures of how closely the investments behave with respect to the reference portfolio. However, they are symmetric measures of distance and dispersion and cannot account for investor aversion for downside rather than upside deviations.

In this contribution we aim at jointly considering the presence of a benchmark and the issue of controlling downside risk. We introduce a set of barriers which accounts for loss/gain preferences of the investor, i.e. the shortfall can be computed with respect to a given level of acceptable losses or with respect to a given desired level of minimum return for the portfolio.

The risk is thus measured by two different components, the first reflects the risk profile of the benchmark; nevertheless, we consider that investors are more concerned with the downside risk and, in particular, with negative deviations from certain reference levels. The resulting portfolio accounts for these two aspect of risk. An indirect measure of risk through the choice of a benchmark and a more direct control on the values of the portfolio through the reference levels. The approach is flexible and allows to easily accounting for different investor preferences. To express risk aversion in portfolio management problems other approaches are possible, mainly based on the definition of a proper utility function and of risk aversion coefficients. They are particularly interesting from a theoretical point of view and have been explored in the literature.

However, in this contribution, we are interested in investigating the connection between the tracking error goal and the control of downside risk both from the point of view of an investor and of a fund manager. To this aim, the use of reference points and the management of risk through shortfalls from the set of specified threshold levels of wealth represent, in our opinion, an easily understandable way of measuring and communicating risk.

The structure of the paper is as follows. In Section 2, we briefly present the contributions in the literature which deal with benchmarking and shortfall control. In Section 3, we present and discuss our model for multiperiod tracking error with shortfall. In Section 4, we present an application of the proposed model and, in order to account for different market conditions, we consider out-of-sample simulation experiments for different periods. Section 5 concludes. 


\section{Literature review}

Different contributions in the literature tackled the issue of benchmarking and tracking error. In a static portfolio selection framework, see, for example, [3][11][24]. For a discussion on the reliability of tracking error as a measure of risk see [26]. While for the use of asymmetric tracking error see [16][20][24].

Among the contributions on dynamic tracking error problems, in continuous or discrete time setting, we refer to [2][8][12][17].

The concern of investors for downside risk has led to the development of a huge stream of financial literature on the use of asymmetric and tail risk measures in portfolio selection problems. There are many contributions which propose the use of alternative risk measures, among them, see for example, [1][7][9][10][15][18][19][25].

We are interested in considering a multiperiod tracking error problem and a discrete time monitoring of the shortfalls below given threshold levels of wealth, we consider both symmetric and asymmetric tracking error measures. To this aim we formulate and solve a multistage stochastic programming problem which provides us with enough flexibility in the formulation of the objective function and of the constraints. To deal with uncertainty in optimization problems other approaches are possible and, in particular, we mention Robust Optimization and its application also to financial optimization problems (see, for example, [4][6][23]).

Dempster et al., in [13][14], tackled the problem of dynamic portfolio management for a pension fund in presence of minimum guarantees. They propose to consider, as objective function, for their multistage stochastic programming problem, the minimization of expected average shortfall and of expected maximum shortfall.

Different contributions in the literature consider the introduction of a shortfall constraint in portfolio management; in particular, for a discussion on the use of shortfall as a risk measure in asset allocation and in static tracking error problem, we refer to [5]. In our problem we consider a discrete monitoring of the portfolio level through the measurement of the shortfalls with respect to a set of reference levels of wealth. This goal is then combined with a tracking error objective and our model can be specified in different ways to account for symmetric and asymmetric distance measures both with respect to the risky benchmark and with respect to the wealth barriers. Moreover, we can allow for a trade-off between the two terms according to the investor's preferences.

\section{Model formulation}

We consider a dynamic tracking error problem and we assume that the investor is interested in tracking the performance of a risky benchmark over time, where the benchmark itself is treated as a stochastic component.

We consider the arborescent formulation of the problem and a scenario tree from $t=0$ (current state) to $T$; we denote with $k_{t}$ a generic node in the event tree at time $t$ and with $\pi_{k_{t}}$ the associated probability. We denote with $y_{k_{t}}$ the value of the managed 
portfolio in each node and with $x_{k_{t}}$ the value of the stochastic benchmark. To control the value of the portfolio we introduce a set of reference levels of wealth, $z_{j t}$ with $j=1, \ldots, J$, which act as thresholds with respect to which we monitor the behavior of the portfolio. In particular, we are interested in monitoring the shortfalls of the portfolio value below each threshold level. The threshold levels are not stochastic but can be time dependent.

The investor can choose among a set of risky assets and a liquidity component. We denote with $q_{i k_{t}}, i=1, \ldots, n$, and $l_{k_{t}}$ the holdings in each asset, while, we use $a_{i k_{t}}$ and $v_{i k_{t}}$ to denote the amounts of risky asset purchased and sold in each node, respectively. The liquidity component absorbs the turnover in the portfolio and accounts also for proportional transaction costs $(t c)$. Moreover, for each node $k_{t}$, we denote with $r_{k_{t}}=\left(r_{1 k_{t}}, \ldots, r_{n k_{t}}, r_{l k_{t}}\right)$ the vector of returns of the risky assets and for the liquidity component for period $[t-1 ; t]$.

We want our model to account both for symmetric and asymmetric distance measures from the risky benchmark and from the reference levels. To this aim we choose to use a mean absolute deviation model (MAD). The mean absolute deviation measure presents many advantages: it leads to a linear optimization problem (see, for example, [21][22][24]), and can be easily separated into positive and negative deviations, allowing for the required flexibility.

In more detail, we define the distance measure of the managed portfolio from the risky benchmark, in node $k_{t}$, as follows

$$
\left|y_{k_{t}}-x_{k_{t}}\right|=\max \left[y_{k_{t}}-x_{k_{t}} ; 0\right]+\max \left[-y_{k_{t}}+x_{k_{t}} ; 0\right]=\theta_{k_{t}}^{+}+\theta_{k_{t}}^{-}
$$

With respect to the threshold levels of wealth $z_{j t}$, with $j=1, \ldots, J$, we are interested in considering only negative deviations from the reference levels and thus we propose to use the following asymmetric distance measure

$$
\left[y_{k_{t}}-z_{j t}\right]^{-}=\max \left[-y_{k_{t}}+z_{j t} ; 0\right]=\gamma_{j k_{t}}^{-}
$$

For a discussion on how the MAD model can be transformed into a linear optimization problem see, for example, [22][24][27].

The resulting multiperiod stochastic programming problem is

$$
\begin{aligned}
& \min \sum_{t=0}^{T}\left[\sum_{k_{t}=K_{t-1}+1}^{K_{t}} \pi_{k_{t}}\left(c^{+} \theta_{k_{t}}^{+}+c^{-} \theta_{k_{t}}^{-}\right)+\sum_{k_{t}=K_{t-1}+1}^{K_{t}} \pi_{k_{t}} \sum_{j=1}^{J} d_{j}^{-} \gamma_{j k_{t}}^{-}\right] \\
& \theta_{k_{t}}^{+}-\theta_{k_{t}}^{-}=y_{k_{t}}-x_{k_{t}} \\
& \gamma_{j k_{t}}^{-} \geq-y_{k_{t}}+z_{j t} \quad j=1, \ldots, J \\
& y_{k_{t}}=l_{k_{t}}+\sum_{i=1}^{n} q_{i k_{t}} \\
& q_{i k_{t}}=\left(1+r_{i k_{t}}\right)\left[q_{i f\left(k_{t}\right)}+a_{i f\left(k_{t}\right)}-v_{i f\left(k_{t}\right)}\right] \\
& l_{k_{t}}=\left(1+r_{l k_{t}}\right)\left[l_{f\left(k_{t}\right)}-\sum_{i=1}^{n}(1+t c) a_{i f\left(k_{t}\right)}+\sum_{i=1}^{n}(1-t c) v_{i f\left(k_{t}\right)}\right]
\end{aligned}
$$




$$
\begin{aligned}
a_{i k_{t}} \geq 0 \quad v_{i k_{t}} \geq 0 \\
q_{i k_{t}} \geq 0 \quad l_{k_{t}} \geq 0 \\
\theta_{k_{t}}^{+} \geq 0 \quad \theta_{k_{t}}^{-} \geq 0 \\
\gamma_{k_{t}}^{-} \geq 0 \\
q_{i 0}=\bar{q}_{i} \quad l_{0}=\bar{l} \\
i=1, \ldots, n \quad k_{t}=K_{t-1}+1, \ldots, K_{t} \quad t=1, \ldots, T
\end{aligned}
$$

where $c^{+}, c^{-}$and $d_{j}^{-}$are positive weights, which may account for a progressive penalization of the deviations or for goal preferences with respect to different threshold levels.

Equation (6) represents the portfolio composition in each node, while equations (7)- (8) describe the dynamics of the assets in the portfolio moving from an ancestor node $f\left(k_{t}\right)$ to a descendent node $k_{t}$. Finally, equation (10) provide the non-negativity conditions on the portfolio composition, ruling out the possibility for short-selling and borrowing, and (13) give the initial portfolio endowments. The objective function of our problem accounts for two different terms. The first is a tracking goal with respect to the risky benchmark while the second term accounts for the shortfalls below the reference levels. The investor is interested in minimizing the distance from the risky benchmark while at the same time limiting the downside risk measured through the shortfalls.

The risk profile of the resulting optimal portfolios takes into account both the connection with the benchmark and the risk aversion for gain or losses lower than a set of specified barriers which are settled by the investors according to their investment goals. The control through reference levels is flexible and presents different advantages in the formulation of the problem. First, barriers are allowed to be time dependent and the risk control can be tailored along the investment horizon. For example, it can be made tighter towards the end of the planning period to account for wealth conservation objectives. Second, the control is introduced in the objective function, rather than in the constraints, in this way we allow for a trade-off with the benchmarking goal and we are able to account for different risk profiles in the investment.

\section{Computational experiments}

In the following we provide an application of the proposed model to real data through an out-of-sample exercise of portfolio management. We assume that our investor is interested in tracking the MSCI Europe Index using a subset of MSCI Style Indexes. To test our model we use a weekly dataset from June 6, 2007 to May 16, 2012. Summary statistics on the Indexes are provided in table 1.

We apply our model simulating the management of the portfolio over a 10-week period using a rolling-horizon procedure. In our experiments we consider two periods to account for different market conditions. The first simulation period ranges 
from December 14, 2011 to February 22, 2012, during this period the benchmark has a significant positive trend, as can be seen from figure 1 . The second simulation period is from February 8, 2012 to April 18, 2012 and the market experiences both huge drops and raises, see figure 2.

For each simulation period, we test different configurations of the objective function in order to analyze the behavior of the optimized tracking portfolio with respect to the benchmark and the threshold levels. In more detail we consider the following settings (in table 2 we summarize the coefficients of the objective function for the portfolio configurations considered in the experiments)

- Portfolio 1a - pure benchmark tracking

- Portfolio $1 \mathrm{~b}$ - benchmark tracking plus a shortfall control

- Portfolio 2a - asymmetric benchmark tracking plus shortfall control

- Portfolio $2 \mathrm{~b}$ - asymmetric benchmark tracking plus enhanced shortfall control

- Portfolio 2c - asymmetric benchmark tracking plus enhanced shortfall control

- Portfolio $3 \mathrm{a}$ - pure shortfall control - one threshold - (no benchmark tracking)

- Portfolio $3 b$ - pure shortfall control - two thresholds - (no benchmark tracking)

The threshold levels used in the simulation periods $(J=2)$ are as follows. For the first period we set $z_{1, t}=1010$ and $z_{2, t}=1020$ constant for all $t=0, \ldots, T$; while we choose $z_{1, t}=970$ and $z_{2, t}=990$, for all $t=0, \ldots, T$, for the second simulation period.

In more detail, the management experiments are carried out as follows. At each step of the simulation we generate a 2-stage scenario tree; we solve the optimization problem (3)-(13) and take the first period optimal decision. The portfolio composition is then evaluated using the true realized returns in the market and the resulting value represents the new endowment for the following period. Clearly, the results of the management experiments depend on the reliability of the generated scenario trees. Many different models can be used to estimate future expected returns and generate the event trees. In this contribution we do not tackle the issue of comparing different models; we propose to generate the scenarios using historical simulation, which assumes only that past returns are good predictors for future behavior without further hypothesis on the return distributions.

In a first analysis we compare a pure tracking error model (portfolio 1a), with portfolio $1 b$ in which we add the controls on the downside deviations from the threshold levels.

A second set of experiments consider only asymmetric distance measures from the risky benchmark and the thresholds levels of wealth. We analyze three different configuration of the objective function where we progressively increase the penalty on the lower barrier, we refer to portfolio $2 a, 2 b$ and $2 c$ in table 2 for the choices of the parameters.

Finally, we consider an experiment in which we drop the tracking goal with respect to the benchmark and consider only the downside penalization of negative deviations from the barriers (see, portfolio $3 a$ and $3 b$, in table 2).

In figure 1 we present the results for the portfolio management experiments for the first period. 


\begin{tabular}{|l|rrrr|}
\hline & Mean & Variance & Skewness & Kurtosis \\
\hline MSCI EUROPE & -0.0014 & 0.0009 & -0.5579 & 1.4773 \\
MSCI EUROPE LG & -0.0004 & 0.0007 & -0.6569 & 1.9806 \\
MSCI EUROPE LV & -0.0021 & 0.0012 & -0.4599 & 1.2498 \\
MSCI EUROPE MG & -0.0010 & 0.0011 & -0.5418 & 1.6296 \\
MSCI EUROPE MV & -0.0024 & 0.0013 & -0.1217 & 0.8914 \\
MSCI EUROPE SMG & -0.0008 & 0.0011 & -0.5507 & 1.7773 \\
MSCI EUROPE SMV & -0.0019 & 0.0013 & -0.1505 & 0.8251 \\
MSCI EUROPE SG & -0.0005 & 0.0012 & -0.5613 & 1.9879 \\
MSCI EUROPE SV & -0.0013 & 0.0013 & -0.1420 & 0.7773 \\
\hline
\end{tabular}

Table 1 Summary statistics MSCI Europe Index and MSCI Europe Style Indexes, weekly data from June 6, 2007 to May 16, 2012.

\begin{tabular}{|l|rrrr|}
\hline & $c^{+}$ & $c^{-}$ & $d_{1}^{-}$ & $d_{2}^{-}$ \\
\hline portfolio 1a & 1 & 1 & 0 & 0 \\
portfolio 1b & 1 & 1 & 1 & 1 \\
portfolio 2a & 0 & 1 & 1 & 1 \\
portfolio 2b & 0 & 1 & 1 & 10 \\
portfolio 2c & 0 & 1 & 1 & 100 \\
portfolio 3a & 0 & 0 & 1 & 0 \\
portfolio 3b & 0 & 0 & 1 & 1 \\
\hline
\end{tabular}

Table 2 Parameters settings for the different portfolio configurations considered in the computational experiments.

We consider, as tracking assets, the following MSCI Style Indexes: LG, LV, SG, SV. From the top graph we can see that the four Style Indexes guarantee a good tracking performance of the index (portfolio $1 a$ ), while, when we introduce a shortfall control for the threshold levels (portfolio $1 b$ ) we sacrifice a potential upside capture. In the middle graph we can observe the portfolio behavior when we consider asymmetric tracking for the risky benchmark and we introduce a progressively higher penalization for the shortfalls (portfolios $2 a, 2 b$ and $2 c$ ). Finally, the bottom graph displays the behavior of the portfolios which account only for shortfall penalization without any tracking component in the objective function.

The same set of experiments has been carried out for the second simulation period. This case is more interesting from the point of view of a downside protection since the index experiences a sharp drop even below the lower threshold.

The same considerations as in the previous set of experiments apply. In particular, the introduction of a shortfall control is effective but is done at the cost of a reduction in the gains when the market rises. Moreover, if we compare portfolios $2 a$ and $1 b$, it is interesting to observe that when we allow for a higher tracking error we can improve the downside protection. From the bottom graph, which displays the behavior of non-tracking portfolios, we can observe that there is an improvement in the downside protection but at a higher cost in terms of upside capture.

The four Style Indexes, we used as tracking assets, allowed us to obtain a good tracking performance in the out-of-sample experiments as it can be seen if we com- 


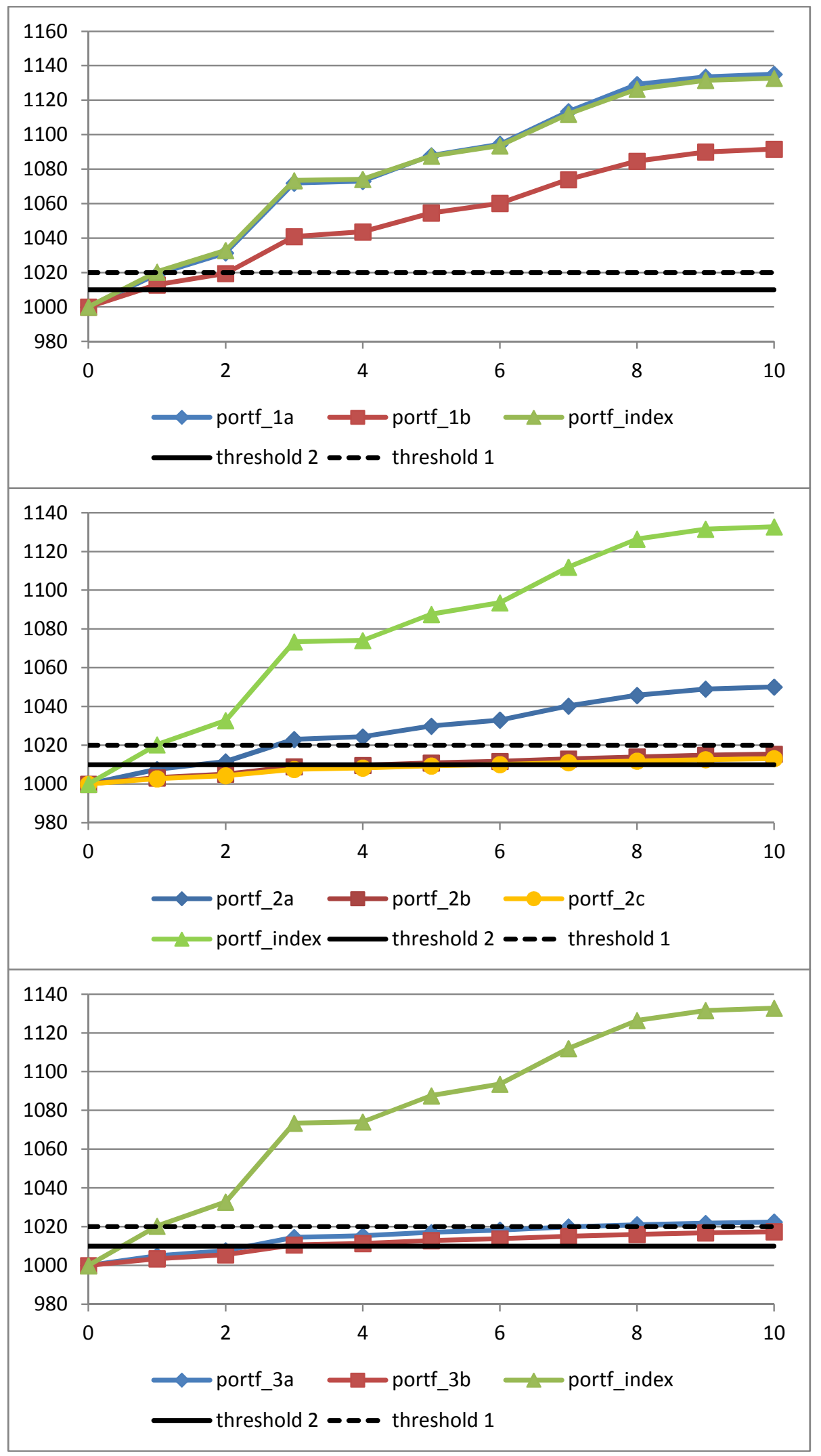

Fig. 1 Comparison between optimized tracking portfolios and benchmark, rolling simulation over 10-week period - December 14, 2011 to February 22, 2012 - two threshold levels $z_{1}=1020$ and $z_{2}=1010$. 


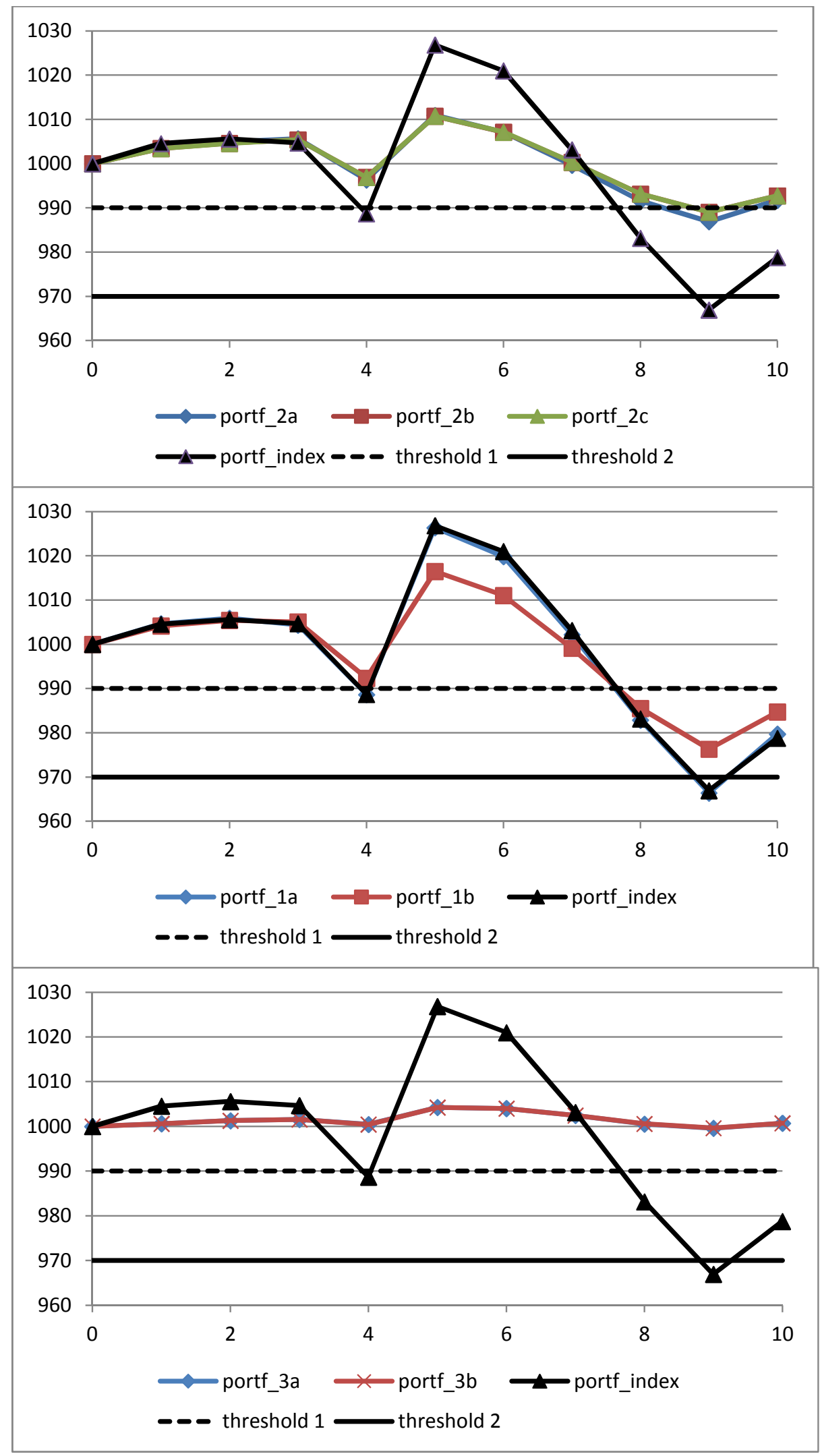

Fig. 2 Comparison between optimized tracking portfolios, rolling simulation over 10-week period - February 8, 2012 to April 18, 2012- two threshold levels $z_{1}=990$ and $z_{2}=970$. 


\begin{tabular}{|l|rrrrrrrrrr|}
\hline & 1 & 2 & 3 & 4 & 5 & 6 & 7 & 8 & 9 & 10 \\
\hline portf 1a & 0.010 & 0.022 & -0.063 & 0.026 & -0.043 & -0.063 & 0.016 & 0.066 & -0.027 & 0.155 \\
portf 1b & -0.040 & 0.024 & 0.055 & 0.325 & -1.425 & 0.032 & 0.572 & 0.634 & 0.715 & -0.363 \\
portf 2a & -0.084 & 0.016 & 0.161 & 0.668 & -2.392 & 0.188 & 1.013 & 1.188 & 1.175 & -0.730 \\
portf 2b & -0.113 & 0.014 & 0.170 & 0.747 & -2.473 & 0.212 & 1.076 & 1.277 & 1.237 & -0.852 \\
portf 2c & -0.113 & 0.014 & 0.170 & 0.748 & -2.472 & 0.213 & 1.076 & 1.277 & 1.237 & -0.854 \\
portf 3a & -0.394 & -0.035 & 0.117 & 1.480 & -3.476 & 0.544 & 1.594 & 1.799 & 1.555 & -1.111 \\
portf 3b & -0.395 & -0.035 & 0.118 & 1.479 & -3.477 & 0.544 & 1.595 & 1.805 & 1.554 & -1.114 \\
\hline
\end{tabular}

Table 3 Percentage Tracking Error with respect to the benchmark, over 10-week period from February 8, 2012 to April 18, 2012, using 4 Style Indexes (LG,LV,SG,SV).

\begin{tabular}{|l|rrrrrrrrrr|}
\hline & 1 & 2 & 3 & 4 & 5 & 6 & 7 & 8 & 9 & 10 \\
\hline portf 1a & 0.037 & 0.021 & 0.033 & 0.032 & 0.019 & 0.006 & 0.049 & 0.098 & 0.033 & 0.083 \\
portf 1b & 0.235 & 0.064 & 0.154 & 0.245 & 0.713 & 0.129 & 0.438 & 0.522 & 0.396 & 0.621 \\
portf 2a & 0.323 & 0.100 & 0.178 & 0.415 & 0.800 & 0.207 & 0.535 & 0.636 & 0.380 & 0.529 \\
portf 2b & 0.302 & 0.098 & 0.167 & 0.361 & 0.717 & 0.189 & 0.504 & 0.529 & 0.309 & 0.358 \\
portf 2c & 0.302 & 0.098 & 0.167 & 0.361 & 0.717 & 0.189 & 0.503 & 0.529 & 0.309 & 0.357 \\
portf 3a & 0.044 & 0.022 & 0.033 & 0.048 & 0.081 & 0.025 & 0.072 & 0.085 & 0.047 & 0.080 \\
portf 3b & 0.044 & 0.022 & 0.034 & 0.050 & 0.079 & 0.025 & 0.071 & 0.085 & 0.048 & 0.078 \\
\hline
\end{tabular}

Table 4 Percentage Tracking Error Volatility with respect to the benchmark, over 10-week period from February 8, 2012 to April 18, 2012, using 4 Style Indexes (LG,LV,SG,SV).

pare portfolios $1 a$ with the Index for both simulation periods. However, in order to analyze the possible improvement in the tracking performances we carried out a further experiment using all the eight Style Indexes.

We computed the Tracking Error (TE) and Tracking Error Volatility (TEV) for both simulation periods for tracking portfolios using as tracking assets the eight Style Indexes. The results, in the two periods, are comparable, and we decide to present only the statistics for the second period, which is more interesting from the point of view of the behavior of the benchmark.

In tables 3 and 4 we report the percentage Tracking Error and percentage Tracking Error Volatility for the analyzed portfolios using four Style Indexes (LG, LV, SG, SV), while tables 5 and 6 presents the same statistics using all the eight Style Indexes (LG, LV, MG, MV, SMG, SMV, SG, SV). Including all the Style Indexes improves the performance but, in our opinion, the tracking results with four Indexes are already satisfying.

The proposed model allows for a great flexibility in the formulation of the objective function and can thus accommodate for different combinations of the tracking and protection goals. Different risk attitude of the investor can be considered in the form of combining a tracking goal with respect to a risky benchmark and introducing a set of desired barriers to control the behavior of the portfolio. 


\begin{tabular}{|l|rrrrrrrrrr|}
\hline & 1 & 2 & 3 & 4 & 5 & 6 & 7 & 8 & 9 & 10 \\
\hline portf 1a & 0.008 & 0.002 & -0.002 & 0.014 & 0.004 & -0.006 & 0.001 & 0.002 & -0.001 & -0.010 \\
portf 1b & -0.078 & 0.028 & 0.075 & 0.300 & -1.359 & 0.087 & 0.589 & 0.644 & 0.737 & -0.536 \\
portf 2a & -0.088 & 0.028 & 0.209 & 0.650 & -2.439 & 0.260 & 1.019 & 1.268 & 1.185 & -0.821 \\
portf 2b & -0.098 & 0.024 & 0.203 & 0.706 & -2.576 & 0.280 & 1.103 & 1.348 & 1.259 & -0.893 \\
portf 2c & -0.099 & 0.024 & 0.203 & 0.706 & -2.576 & 0.280 & 1.103 & 1.348 & 1.261 & -0.894 \\
portf 3a & -0.399 & -0.038 & 0.126 & 1.477 & -3.458 & 0.545 & 1.598 & 1.780 & 1.558 & -1.172 \\
portf 3b & -0.405 & -0.037 & 0.126 & 1.477 & -3.462 & 0.546 & 1.603 & 1.774 & 1.559 & -1.171 \\
\hline
\end{tabular}

Table 5 Percentage Tracking Error with respect to the benchmark, over 10-week period from February 8, 2012 to April 18, 2012, using 8 Style Indexes (LG,LV,MG,MV,SMG,SMG,SG,SV).

\begin{tabular}{|l|rrrrrrrrrr|}
\hline & 1 & 2 & 3 & 4 & 5 & 6 & 7 & 8 & 9 & 10 \\
\hline portf 1a & 0.017 & 0.005 & 0.013 & 0.011 & 0.027 & 0.013 & 0.022 & 0.054 & 0.023 & 0.078 \\
portf 1b & 0.219 & 0.070 & 0.148 & 0.261 & 0.655 & 0.138 & 0.462 & 0.502 & 0.353 & 0.560 \\
portf 2a & 0.332 & 0.103 & 0.204 & 0.459 & 0.872 & 0.201 & 0.543 & 0.559 & 0.352 & 0.394 \\
portf 2b & 0.321 & 0.102 & 0.187 & 0.424 & 0.712 & 0.191 & 0.493 & 0.471 & 0.288 & 0.319 \\
portf 2c & 0.321 & 0.101 & 0.187 & 0.423 & 0.713 & 0.190 & 0.492 & 0.472 & 0.287 & 0.320 \\
portf 3a & 0.069 & 0.015 & 0.031 & 0.042 & 0.105 & 0.022 & 0.060 & 0.110 & 0.046 & 0.063 \\
portf 3b & 0.066 & 0.014 & 0.032 & 0.040 & 0.113 & 0.021 & 0.061 & 0.114 & 0.045 & 0.071 \\
\hline
\end{tabular}

Table 6 Percentage Tracking Error Volatility with respect to the benchmark, over 10-week period from February 8, 2012 to April 18, 2012, using 8 Style Indexes (LG,LV,MG,MV,SMG,SMG,SG,SV).

\section{Concluding remarks}

In this contribution we propose a multiperiod tracking error problem which can account for shortfall control using a sequence of references levels for wealth. The use of thresholds to define the goals for the portfolio management problem is intuitive for the investor and avoids the choice of a proper utility function and the definition of risk attitude/tolerance parameters. The proposed model allows to consider asymmetric tracking measures and in particular to penalize only downside deviations from the reference wealth levels. The computational experiments discussed show the trade-off between the possibility of upside capture and the control on downside risk. The role of the number of thresholds and the choice of a progressive penalization could be further investigated.

The authors thank dott. Fabio Lanza for the research assistance in the computational experiments.

\section{References}

1. Alexander, G.J., Baptista, A.M.: Portfolio selection with a drawdown constraint. Journal of Banking and Finance 30 (2006) 3171-3189 
2. Barro, D., Canestrelli, E.: Tracking error: a multistage portfolio model. Annals of Operations Research, 165(1) (2009) 44-66

3. Basak, S., Shapiro, A., and Tepla, L.: Risk management with benchmarking. Working Paper Series Asset Management SC-AM-03-16, Salomon Center for the study of financial institutions, NYU (2003)

4. BenTal, A., Nemirovski, A.: Robust optimization - methodology and applications. Mathematical programming, Ser. B 92 (2002) 453-480

5. Bertsimas, D., Lauprete, G.J., Samarov, A.: Shortfall as a risk measure: properties, optimization and applications. Journal of Economic Dynamics and control 28 (2004) 1353-1381

6. Bertsimas, D., Sim, M.: The price of robustness. Operations Research 52 (2004) 35-53

7. Biglova, A., Ortobelli, S., Rachev, S., Stoyanov, S.: Different approaches to risk estimation in portfolio theory. The Journal of Portfolio Management (2004) 103-112

8. Browne, S.: Beating a moving target: optimal portfolio strategies for outperforming a stochastic benchmark. Finance and Stochastics 3 (1999) 255-271

9. Chekhlov, A., Uryasev, S., Zabarankin, M.: Drawdown measure in portfolio optimization. International Journal of Pure and Applied Finance 8(1) (2005) 13-58

10. Cuoco, D., He, H., Isaenko, S.: Optimal dynamic trading strategies with risk limits. Operations Research 56(2) (2008) 358-368

11. Dembo, R., Rosen, D.: The practice of portfolio replication, a practical overview of forward and inverse probems. Annals of Operations Research 85 (1999) 267-284

12. Dempster, M.H.A., Thompson, G.W.P.: Dynamic portfolio replication using stochastic programming. In M.H.A. Dempster (Ed.), Risk Management: value at risk and beyond (2002) 100-128. Cambridge University Press.

13. Dempster, M.A.H., Germano, M., Medova, E.A., Rietbergen, M.I., Sandrini, F., and Scrowston, M.: Managing guarantees. The Journal of Portfolio Management 32(2) (2006)

14. Dempster, M.A.H., Germano, M., Medova, E.A., Rietbergen, M.I., Sandrini, F., and Scrowston, M.: Designing minimum guaranteed return funds. Quantitative Finance 7(2) (2007) 245256

15. Ebert, U.: Measures of downside risk. Economics Bulletin 4(16) (2005) 1-9

16. Franks, E.C.: Targeting excess-of-benchmark returns. The Journal of Portfolio Management 18 (1992) 6-12

17. Gaivoronski, A., Krylov, S., van der Vijst, N.: Optimal portfolio selection and dynamic benchmark tracking. European Journal of Operational Research 163 115-131

18. Ibrahim, K., Kamil, A.A., Mustafa, A.: Portfolio selection problem with maximum deviation measure: a stochastic programming approach. International Journal of Mathematical Models and Methods in Applied Sciences 1(2) (2008) 123-129

19. Jansen, D.W., Koedijk, K.G., de Vries, C.G.: Portfolio selection with limited downside risk. Journal of Empirical Finance 7 (2000) 247-269

20. King, A.J.: Asymmetric risk measures and tracking models for portfolio optimization under uncertainty. Annals of Operations Research 45 (1993) 165-177

21. Konno, H., Yamazaki, H.: Mean absolute deviation portfolio optimization model and its applications to Tokyo stock market. Management Science 37 (1991) 519-531

22. Michalowski, W., Ogryczak, W.: Extending the MAD portfolio optimization model to incorporate downside risk aversion. Naval Research Logistics, 48 (2001) 185-200

23. Quaranta, A.G., Zaffaroni, A.: Robust optimization of conditional value at risk and portfolio selection. Journal of Banking and Finance 32 (2008) 2046-2056

24. Rudolf, M., Wolter, H-J., Zimmermann, H.: A linear model for tracking error minimization. Journal of Banking and Finance 23 (1999) 85-103

25. Rockafellar, R.T., Uryasev, S.: Optimization of Conditional Value at risk. Journal of Risk 2 (2000) 21-41

26. Scowcroft, A., Sefton, J.: Do tracking errors reliably estimate portfolio risk? Journal of Asset Management 2(3) (2000) 205-222

27. Shanno, D.F., Weil, R.L.: Linear Programming with Absolute-Value Functionals. Operations Research 19(1) (1971) 120-124 